\title{
Cyclic GMP signaling in rat urinary bladder, prostate, and epididymis: tissue-specific changes with aging and in response to Leydig cell depletion
}

\author{
Dieter Müller, Amal K Mukhopadhyay ${ }^{1}$, Michail S Davidoff ${ }^{2}$ and Ralf Middendorff \\ Institute of Anatomy and Cell Biology, Justus-Liebig-University, Aulweg 123, 35385 Giessen, Germany, ${ }^{1}$ ELGA \\ Biotech, Elbgaustrasse 71, 22523 Hamburg, Germany and ${ }^{2}$ Institute of Anatomy, University of Hamburg, 20246 \\ Hamburg, Germany \\ Correspondence should be addressed to D Müller; Email: hans-dieter.mueller@anatomie.med.uni-giessen.de
}

\begin{abstract}
Aging of the male reproductive system leads to changes in endocrine signaling and is frequently associated with the emergence of prostate hyperplasia and bladder dysfunctions. Recent reports highlight prostate and bladder as promising targets for therapeutic interventions with inhibitors of the cyclic GMP (cGMP)-degrading phosphodiesterase 5 (PDE5). However, the cGMP signaling system in these organs is as yet poorly characterized, and the possibility of age-related alterations has not been addressed. This study investigates key proteins of cGMP pathways in bladder, prostate, and epididymis of young (3 months) and old (23-24 months) Wistar rats. Local differences in the abundance of PDE5, soluble guanylyl cyclase (sGC) and particulate guanylyl cyclases (GC-A, GC-B), endothelial nitric oxide synthase, and cGMP-dependent protein kinase I (PRKG1 (cGKI)) revealed pronounced tissue-specific peculiarities. Although cGMP-generating enzymes were not affected by age in all organs, we recognized age-related decreases of PDE5 expression in bladder and a selective diminishment of membrane-associated PRKG1 in epididymis. In disagreement with published data, all cGMP pathway proteins including PDE5 are poorly expressed in prostate. However, prostatic PRKG1 expression increases with aging. Androgen withdrawal during temporary Leydig cell elimination induced a massive ( $>12$-fold) upregulation of PRKG1 in prostate but not in other (penis and epididymis) androgen-dependent organs. These findings identify PRKG1 as a key androgen-sensitive signaling protein in prostate of possible importance for growth regulation. The elucidated effects may have significance for age-associated pathologies in the male lower-urinary tract.

Reproduction (2011) 142 333-343
\end{abstract}

\section{Introduction}

Signal transduction pathways using cyclic GMP (cGMP) as second messenger regulate diverse physiological functions such as smooth muscle contractility, neurotransmission, and cell growth/proliferation (Hofmann et al. 2006). The benefit of agents that inhibit phoshodiesterase 5 (PDE5, a cGMP-degrading enzyme) for treatment of erectile dysfunction and pulmonary hypertension (Ghofrani et al. 2006) underscores the significance of cGMP signaling and indicates broad clinical potential. In most tissues, cellular accumulations of cGMP are induced by either natriuretic peptide (NP) membrane receptors with guanylyl cyclase (GC) activity (referred to as GC-A and GC-B) and/or the nontransmembrane protein soluble GC (sGC). GC-A (also known as NPR-A or NPR1) is activated by the cardiac hormones atrial NP (ANP, also known as NPPA) and B-type NP (also known as NPPB), whereas GC-B (NPR-B or NPR2) acts as specific receptor for a third peptide,
C-type NP (CNP, also known as NPPC; Kuhn 2009). Stimulation of $\mathrm{SGC}$ is evoked by nitric oxide (NO) binding, and one important and widely distributed generator of $\mathrm{NO}$ is the enzyme endothelial $\mathrm{NO}$ synthase (NOS3, also known as eNOS; Alderton et al. 2001). Accumulations of cGMP are under the control of cGMPdegrading PDEs, with PDE5 representing a crucially implicated subtype (Ghofrani et al. 2006). Cellular effects are finally induced by cGMP target proteins. cGMPmediated activation of the CGMP-dependent protein kinase I (PRKG1) plays a pivotal role in all three physiological roles denoted above (Hofmann et al. 2006).

Recent findings raised attention to cGMP-regulated functions and possible treatment options for PDE5 inhibitors in bladder, prostate, and epididymis (Andersson et al. 2007, Dimitriadis et al. 2009, Sandner et al. 2009, Wang 2010). Local roles for cGMP signaling comprise the regulation of muscle contractions and micturition sensations in the bladder (Persson et al. 2000, Andersson \& Arner 2004, Sandner et al. 2009, Caremel et al. 2010), 
the relaxation of stromal smooth muscle and control of cell proliferation in the prostate (Gradini et al. 1999, Sandner et al. 2009, Fibbi et al. 2010, Zenzmaier et al. 2010), and regulation of peristalsis and secretory epithelial cell functions in the epididymis (Mewe et al. 2006, Shum et al. 2008). Although certain factors implicated in cGMP pathways have been demonstrated by immunohistochemical, RT-PCR, and functional approaches, the cGMP signaling system in these organs is still poorly characterized. Thus, one goal of the present investigation was to examine and substantiate the local expression of cGMPgenerating enzymes and other key components of cGMP pathways. Organs (e.g. lung and brain) where molecular and functional activities of the cGMP signaling system have been more thoroughly investigated before were co-analyzed.

Growing evidence indicates that aging can lead to changes in the expression and activity of cGMP signaling proteins with significant impact for organ functions. For example, reduced neuronal cGMP levels, mainly caused by aging-associated increases in CGMP-degrading PDEs, have been linked to impaired synaptic function and memory (Domek-Łopacińska \& Strosznajder 2010). Age-related alterations in cGMP pathways of functional significance have also been observed in vascular (Stice et al. 2009) and penile (Sampson et al. 2007) tissues. The molecular basis for the frequent development of benign prostatic hyperplasia, prostate cancer, and bladder dysfunctions in older men remains poorly understood (Love et al. 2009, Goldstein et al. 2010, Wang 2010). To facilitate detection of possible age-related changes within the cGMP signaling system, the expression of cGMP pathway proteins in bladders, prostates, and epididymides derived from either young adult ( 3 months old) or old (23-24 months) Wistar rats was compared. To examine androgen-dependent effects, we used an experimental model in which injection of the compound ethane dimethane sulfonate (EDS) into adult rats evokes a temporary elimination of the testosteroneproducing Leydig cell population (Molenaar et al. 1985, Davidoff et al. 2004).

Our investigations revealed an unexpected low abundance of all cGMP signaling proteins including PDE5 in the prostate, elucidated distinct organ- and pathway-specific age-related changes, and uncovered a hitherto unrecognized androgen-dependent suppression of PRKG1 expression in prostatic tissue.

\section{Results}

\section{Pronounced tissue-specific expression patterns of cGMP pathway proteins}

Initial studies served to characterize by western blot analyses the relative abundance in bladder, prostate, and epididymis of proteins implicated in cGMP signaling. For comparison, expression levels in three reference organs (lung, brain, and testis) were co-analyzed. Specifically, we examined the three cGMP-generating proteins GC-A, GC-B, and sGC; the NO-producing enzyme NOS3; the cGMP-degrading enzyme PDE5; and the cGMP target protein PRKG1. As the non-transmembrane proteins SGC, NOS3, PDE5, and PRKG1 may have either soluble (cytosolic) or particulate (membrane) cellular locations, and considering that the relative amount in particulate fractions is indicative of membrane-localized signaling microdomains (Takimoto et al. 2009, Müller et al. 2010a) the concentrations of these proteins were monitored in both cytosolic $(\mathrm{C})$ and membrane $(\mathrm{M})$ fractions.

Data obtained (Fig. 1A) revealed marked tissue-specific characteristics. Consistent with expected features of local cGMP production in the reference organs, the lung was distinguished by highest abundance of all three (GC-A, GC-B, and sGC) cGMP-generating enzymes, the brain by a conspicuous predominance of SGC and the testis by peculiar high GC-A levels, reflecting strong expression of this receptor in seminiferous tubules (Müller et al. 2004). Comparison between bladder and epididymis demonstrated higher abundance of sGC in the former and predominating levels of particulate GCs in the latter organ. Note that the size heterogeneity detectable in the case of GC-A and GC-B is explained by tissue-specific differences in glycosylation (Müller et al. 2002, 2010b). The study revealed that the urinary bladder is an outstanding expression site for NOS3, PDE5, and PRKG1, and particularly, high amounts of PDE5 and PRKG1 are membrane-associated in this tissue.

Intriguingly, the prostate was marked by extraordinarily poor expression levels of all enzymes implicated in cGMP signaling pathways (Fig. 1A, framed). Protein staining of the blots confirmed that equal amounts of total protein were analyzed from each tissue (Supplementary Fig. 1, see section on supplementary data given at the end of this article, demonstrates the staining of the membrane and cytosolic protein fractions employed). To visualize the (poor) expression of PDE5 in prostate and epididymis, higher amounts of protein were analyzed (Supplementary Fig. 2A, see section on supplementary data given at the end of this article). The exceptionally low expression of PRKG1 in prostate was confirmed by a comparative analysis between six organs of the male reproductive/lower-urinary tract including seminal vesicle and penis (Supplementary Fig. 2B).

Further studies showed that the exceptionally low abundance of cGMP signaling proteins in prostate represents a pathway-specific phenomenon, because levels of unrelated signaling proteins such as glycogen synthase kinase- $3 \alpha$ and $\beta$ (GSK3A and GSK3B; Fig. 1B) or phosphatase and tensin homolog (PTEN; data not shown) were not reduced in a prostate-selective manner. Densitometric analyses (Fig. 1C) served to indicate the relative expression of the cGMP pathway proteins among bladder, prostate, and epididymis. 
A

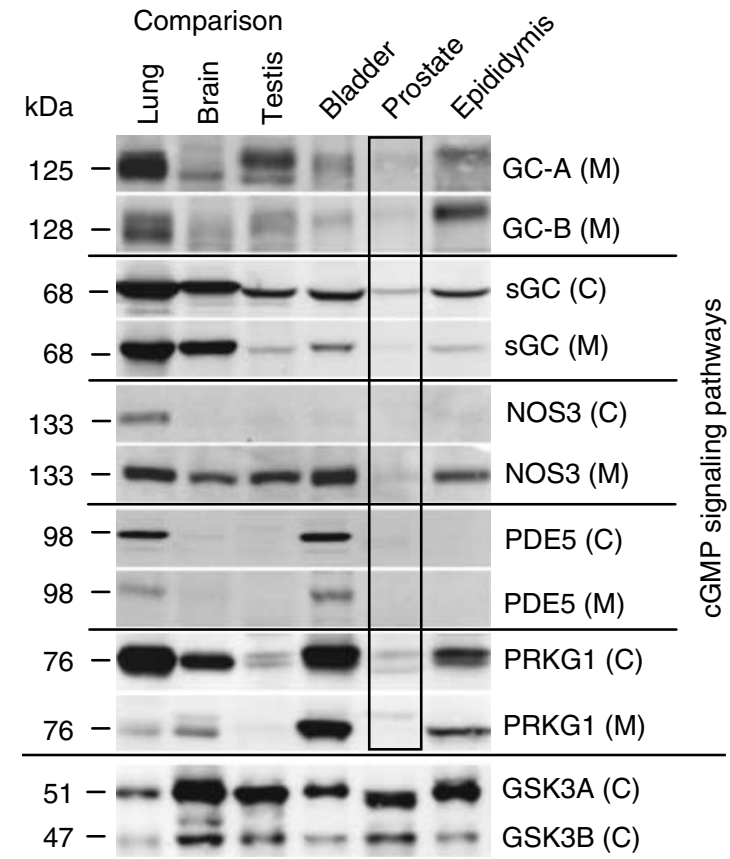

C

Relative abundance (\%) in

bladder $(B)$, prostate $(P)$, and epididymis $(E)$
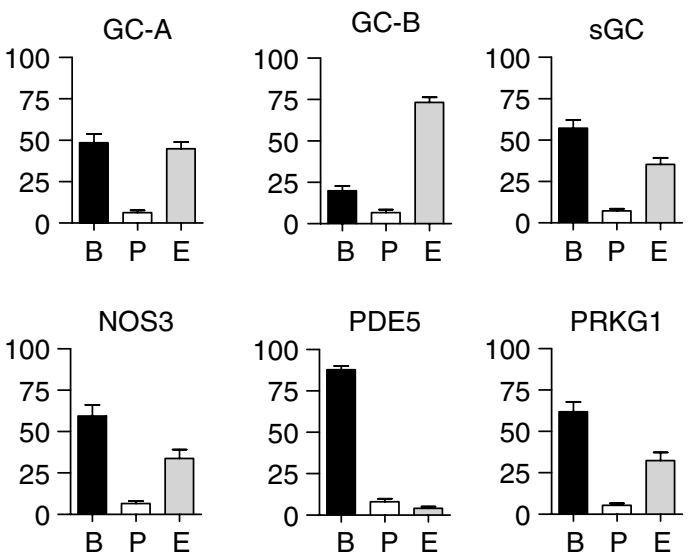

Figure 1 Comparative analysis of key proteins involved in cGMP signaling among lung, brain, testis, bladder, prostate, and epididymis. (A) Equal amounts $(70 \mu \mathrm{g})$ of membrane $(\mathrm{M})$ or cytosolic (C) proteins from the rat tissues indicated were analyzed by immunoblotting using antibodies against GC-A, GC-B, sGC (subunit $\beta 1$ ), NOS3, PDE5, and PRKG1. Apparent molecular masses $(\mathrm{kDa})$, calculated from the migration of marker proteins, are indicated in the left. The heterogeneous sizes of GC-A and GC-B are explained by tissuespecific modes of glycosylation. Data shown are representative of analyses performed with tissues from seven 3-month-old Wistar rats. (B) Blots used in A were stripped and re-exposed to antibodies against GSK3A/B. (C) Relative abundance of cGMP signaling proteins in bladder, prostate, and epididymis tissue. Data represent mean \pm s.E.M. from densitometric quantifications of experiments $(n=4)$ performed with membrane (GC-A, GC-B, and NOS3) or cytosolic (sGC, PDE5, and PRKG1) fractions from different animals.

\section{Aging does not affect cGMP-generating enzymes}

To examine whether aging leads to changes in cGMPgenerating proteins, we compared the expression levels of GC-A, GC-B, and sGC among organs from young adult (y, 3 months) and old (o, 23-24 months) Wistar rats. These studies, performed with tissues from at least ten animals each, did not show any aging-associated alterations in bladder, prostate, and epididymis. Figure 2A provides a representative analysis, carried out with membrane and cytosolic (sGC) protein fractions from two rats of each age group.

To assess the functional activity of the two NP receptors, membranes from bladder and epididymis were exposed to ANP (the GC-A ligand) or CNP (the GC-B ligand), and the amounts of cGMP produced after 12 min were determined. The results (Fig. 2B) revealed the absence of age-related effects on GC-A and GC-B activity in the two organs examined. They also provided evidence that membrane-associated cGMP production in the epididymis is predominantly realized by CNP/GC$B$ rather than ANP/GC-A signaling. The peptide specificity and time dependency of cGMP production by epididymis membranes is demonstrated (Supplementary Fig. 3, see section on supplementary data given at the end of this article). Analogous experiments performed with prostate membranes (data not shown) revealed much lower basal cGMP ( $<3$ pmol cGMP/mg membrane protein) levels, and both ANP and CNP were unable to significantly enhance the production of cGMP.

\section{Age-related decline in PDE5 abundance in bladder}

To look for potential age-dependent changes of other proteins involved in CGMP signaling, comparative immunoblot analyses were carried out for NOS3, PDE5, and PRKG1. These studies revealed that the cytosol of aged bladders contains markedly reduced amounts of PDE5 (Fig. 3A; cytosolic fractions from five animals per age group were analyzed). As generally used in all immunoblot analyses (see section Materials and Methods and Supplementary Fig. 4, see section on supplementary data given at the end of this article), equal protein loading was confirmed by Ponceau staining of the blot prior to antibody exposure. Co-analysis of different protein amounts (three lanes to the right) from one cytosolic extract of a young animal proved i) that the anti-PDE5 antibody properly recognized differences in the level of its target protein and ii) that Ponceau staining provides a reliable loading control. Further analyses of pooled cytosolic fractions ( 1 and 2; from five animals each) verified the aging-associated decrease in PDE5 expression (Fig. 3B). Examinations of PRKG1 and sGC, performed consecutively on the PDE5 antibody-exposed blot, corroborated a PDE5-specific downregulation. Analogous assays, carried out with pooled membrane fractions, also showed age-dependent decreases in PDE5 


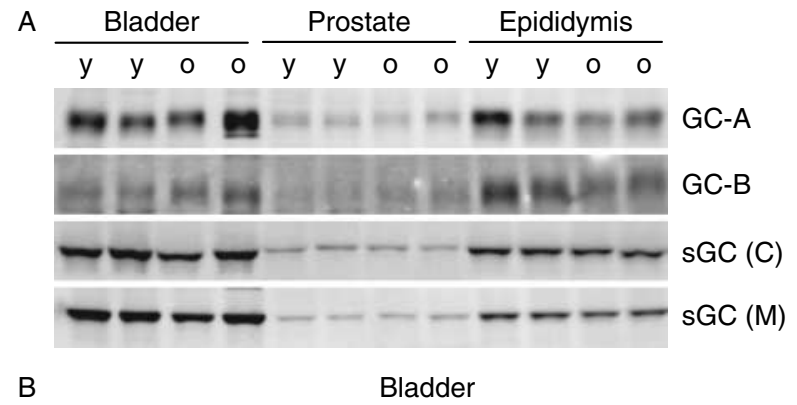

B

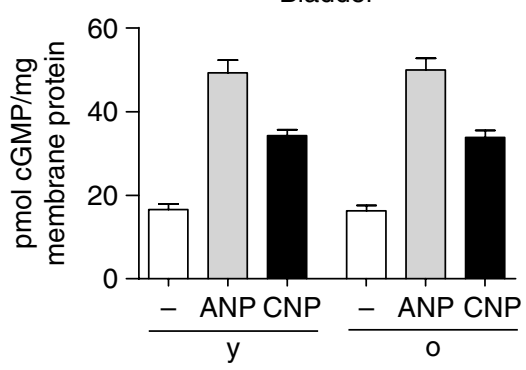

C

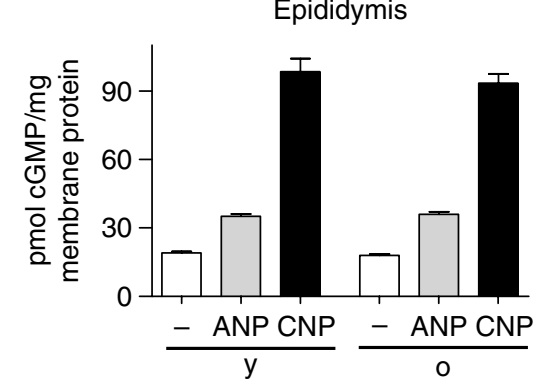

Figure 2 Examination of GC-A, GC-B, and SGC in bladder, prostate, and epididymis from young (y) and old (o) rats. (A) Expression levels of GC-A and GC-B were analyzed by immunoblotting of equal amounts $(60 \mu \mathrm{g})$ of membrane protein. Protein levels of sGC were assessed in both membrane (M; $100 \mu \mathrm{g}$ protein) and cytosolic (C; $60 \mu \mathrm{g})$ fractions. The results showing analyses of tissues from two animals per group, were representative of examinations performed with more than ten animals of each age. Equal protein loading was controlled by Ponceau staining. (B) Equal amounts (10 $\mu$ g protein) of bladder membranes from young or old animals were incubated in either the absence $(-)$ or the presence $(1 \mu \mathrm{M})$ of ANP or CNP for $12 \mathrm{~min}$, and values of cGMP produced were determined by ELISA. Data shown ( \pm s.E.M.) are based on quadruplicate measurements of three experiments performed with membrane proteins from different animals. (C) Analogous experiments as in B were carried out with epididymis membranes.

(but not PRKG1 and sGC) levels (Fig. 3C). Supporting PDE5-specific effects, NOS3 concentrations were not different between young and old (Fig. 3D). Densitometric quantifications (Fig. 3E) revealed a highly significant $(P<0.0001)$ diminishment (by $\sim 45 \%)$ of cytosolic PDE5 protein levels in the rat urinary bladder with aging.

\section{Specific decreases in membrane-associated PRKG1 levels in the aged epididymis}

We did not find any differences in sGC or PDE5 levels between young and old in the epididymis (data not shown). However, comparative analyses consistently demonstrated lower levels of PRKG1 in epididymis membrane fractions from old compared with young animals (Fig. 4A; $a$ and $b$ represent examinations of different animals; $c$ shows the protein staining of b). The same blot (b) was used, after stripping, for analyses of GC-B and SGC expression. Neither the levels of the transmembrane protein GC-B nor those of the membrane-associated sGC were different between young and old (d and e). Importantly, age-dependent decreases in PRKG1 were not evident when cytosolic fractions from the same animals were examined (Fig. 4B). Also, PDE5 levels remained unaffected by aging (Fig. 4B). Thus, these findings revealed a highly significant $(* P<0.0001)$ and specific diminution (by nearly $50 \%$ ) of membranelocalized PRKG1 in the aged epididymis (Fig. 4C).

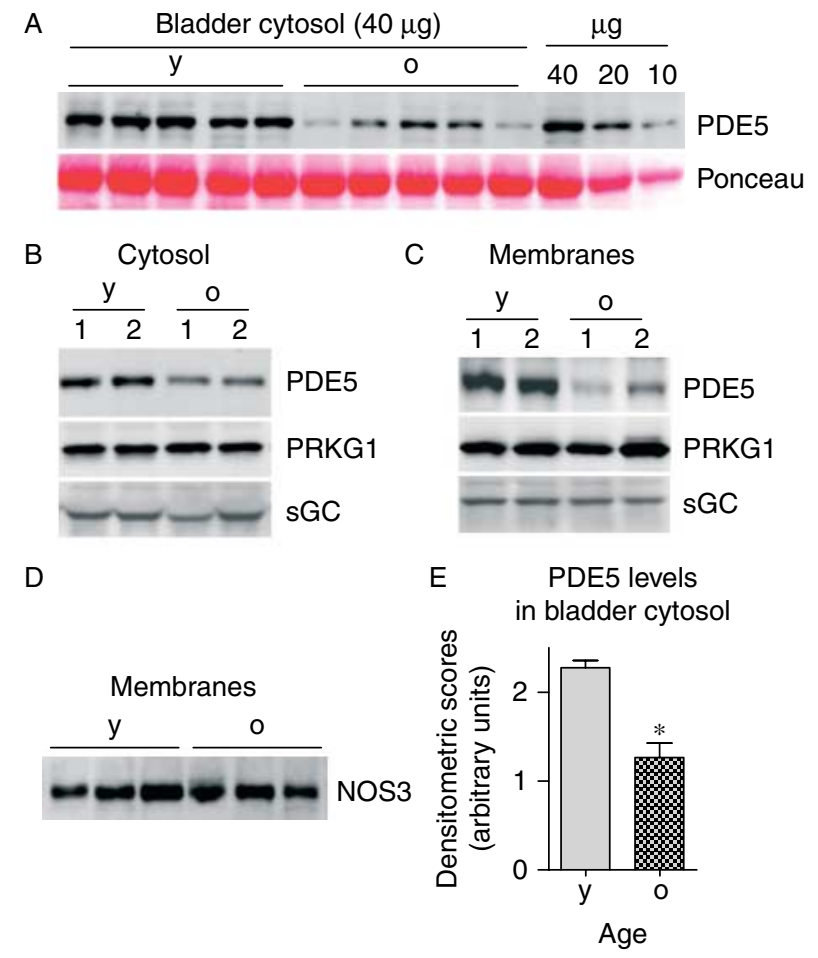

Figure 3 Age-related decrease of PDE5 abundance in bladder. (A) Immunoblot for PDE5 with bladder cytosolic protein fractions (40 $\mu \mathrm{g})$ from five young (y) and old (o) animals each. Co-analyses of different protein amounts $(40,20$, and $10 \mu \mathrm{g}$, derived from a young animal) and the protein staining of the blot (Ponceau) are shown. (B) Pooled cytosolic protein fractions from $2 \times 5$ young and old rats each ( 1 and 2 ) were analyzed for PDE5 expression by immunoblotting. The same blot was used, after stripping, to examine consecutively PRKG1 and sGC levels. (C) Membrane fractions, pooled in analogy to $\mathrm{B}$, were examined for the expression of PDE5, PRKG1, and sGC. (D) Equal amounts of bladder membranes from either young (y) or old (o) animals were analyzed for NOS3 expression. (E) Densitometric quantification of the age-related downregulation of PDE5 in bladder. The analyses of cytosolic fractions from ten individuals per group revealed significantly $\left({ }^{*} P<0.0001\right)$ lowerprotein levels in old animals. Values are mean \pm s.E.M. In all experiments (A-E), equal protein loading was confirmed by Ponceau staining as demonstrated exemplarily in Supplementary Fig. 4, see section on supplementary data given at the end of this article. 
A

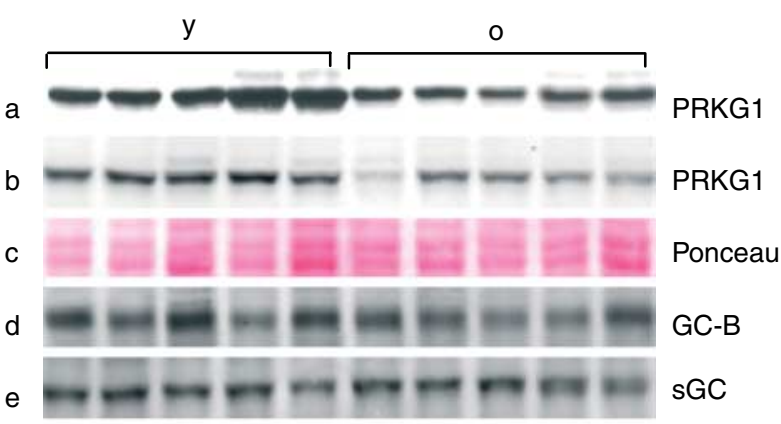

B

Epididymis cytoso

y

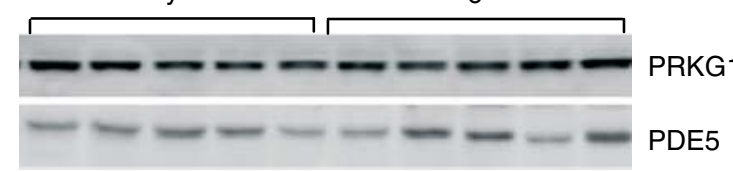

C

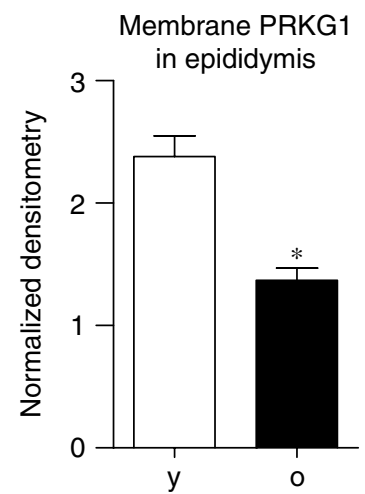

Figure 4 Decline of membrane-associated PRKG1 levels with aging in the epididymis. (A) Membrane fractions ( $60 \mu$ g protein) of epididymides from five young (y) and old (o) animals each were analyzed for PRKG1 expression by immunoblotting (a). Analogous experiment performed with epididymides from other animals (b). Protein staining (Ponceau) of the blot presented in $\mathrm{b}$ is shown in panel c. After stripping, this protein blot was used to monitor the expression of GC-B (d) and sGC (e). (B) Immunoblots for PRKG1 and PDE5 carried out with cytosolic protein fractions (40 or $120 \mu$ g protein respectively). Equal protein loading was controlled. (C) Densitometric quantification of membranelocalized PRKG1 levels in epididymides from young and old animals $\left(n=10\right.$ each). The age-related diminution is highly $\left({ }^{*} P<0.0001\right)$ significant. Values are mean \pm s.E.M.

\section{Age-related increase in PRKG1 expression levels in the prostate}

Analogous assays, performed with elevated $(\geq 100 \mu \mathrm{g})$ protein amounts, served to examine potential agingassociated changes in the prostate. These studies revealed that PRKG1 levels are markedly higher in cytosolic protein fractions from old compared with young animals (Fig. 5A). As evidenced, using the same blots for examination of PDE5, expression levels of the cGMP-specific PDE were not found to be affected by aging (Fig. 5A). Certain age-related increases in PRKG1 were also detectable in prostate membranes (Fig. 5B).
Quantitative evaluations provided evidence for a highly significant $(P<0.0001)$ increase $(1.7$-fold $)$ in PRKG1 levels in the aged prostate (Fig. 5C).

\section{Elucidation of a prostate-specific androgen regulation}

Considering the pronounced androgen dependency of prostate tissue (Love et al. 2009), it was of interest to investigate the expression of cGMP pathway proteins in this tissue under conditions of androgen ablation. To address this item, we made use of an animal model in which a single injection of EDS into mature rats transiently and specifically eliminates the testosteroneproducing Leydig cell population (Molenaar et al. 1985). As detectable by analyses of the Leydig cell marker protein CYP11A1 (Cyt P450scc) in testis extracts (Fig. 6A), Leydig cells are destructed between days 2 and 14 after EDS injection and re-appear some days later (Davidoff et al. 2004). Corresponding immunoblots performed with cytosolic proteins of the prostates revealed a striking upregulation of PRKG1 at days 7

A

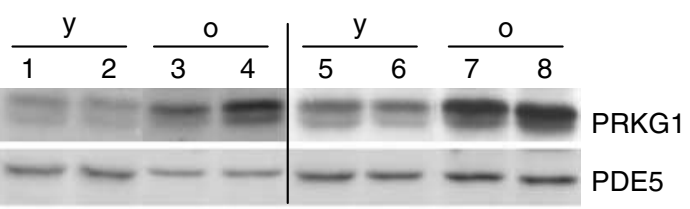

B

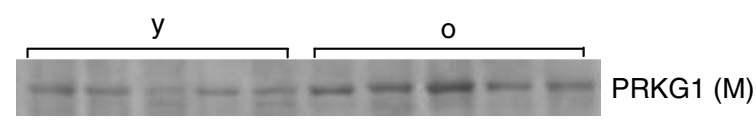

C

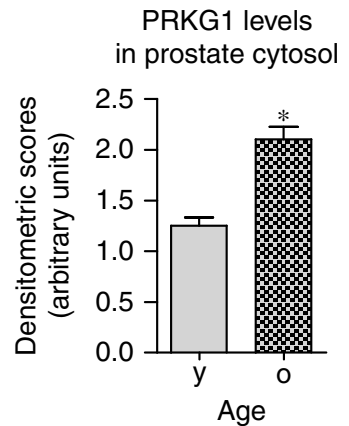

Figure 5 Aging-associated increase in PRKG1 in the prostate. (A) Different pools of prostates, collected from either young ( $y$; 1 and 2 ) or old ( $0 ; 3$ and 4) animals, were homogenized, and cytosolic protein fractions were prepared by parallel operations (lanes 5-8 represent proteins from an independent replication with tissues from other animals). Equal amounts (100 $\mu \mathrm{g})$ of proteins were analyzed via western blotting, and the same blots were used to visualize consecutively PDE5 immunoreactivity. (B) Immunoblot analyses with prostate membranes from five young (y) and old (o) animals indicate age-related increases in membrane-associated (M) PRKG1. (C) Densitometric quantification of cytosolic PRKG1 levels $(n=8$ rats per age group) reveals significant $\left({ }^{*} P<0.0001\right)$ increases with aging (values are mean \pm S.E.M.). In all experiments $(A-C)$, equal protein loading was confirmed by Ponceau staining as demonstrated exemplarily in Supplementary Fig. 4, see section on supplementary data given at the end of this article. 
and 14 after EDS (Fig. 6A). The onset of PRKG1 upregulation was delayed in comparison to the disappearance of CYP11A1 (see days 2 and 3), and the effect was rapidly and completely abrogated after Leydig cell regeneration (day 20).

Analyses of further animals, covering days $1-47$ after EDS, confirmed the massive and temporary elevation of PRKG1 expression in the prostate (Fig. 6B). To evaluate the specificity of this effect, analogous immunoblot experiments were performed with antibodies against
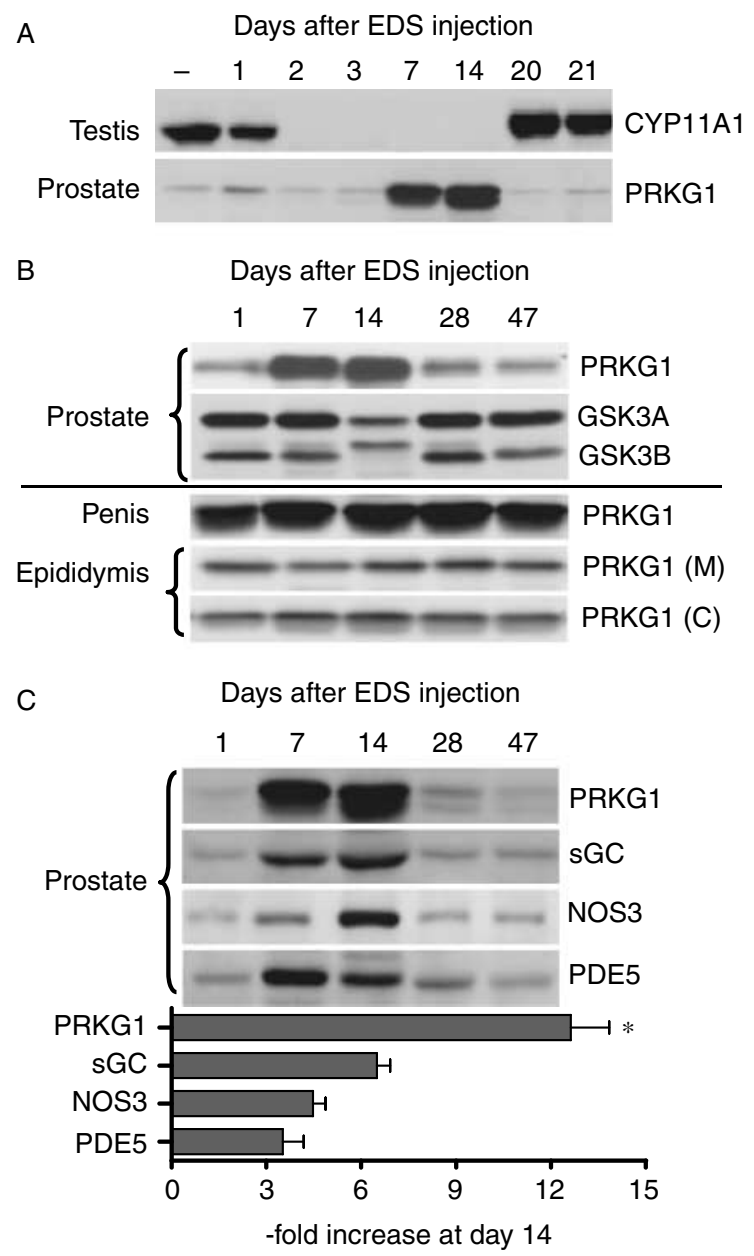

Figure 6 Effects elicited by Leydig cell depletion. (A) Equal amounts of membrane (testis) or cytosolic (prostate) proteins from EDS-treated or untreated (-) rats were analyzed for expression of the Leydig cell marker protein CYP11A1 and PRKG1 by immunoblotting as indicated. (B) Immunoblot analyses of different kinases in prostate, penis, and epididymis tissue derived from rats at different days after EDS injection. Assays were performed with cytosolic protein fractions. Both cytosolic (C) and membrane (M) proteins were analyzed in the case of epididymis. (C) Equal amounts of cytosolic (PRKG1, sGC, and PDE5) or membrane (NOS3) proteins from prostates of EDS-treated rats were analyzed by immunoblotting. The lower panel shows a densitometric quantification based on four experiments. Increases at day 14 after EDS were calculated in relation to the scores assessed for day 1 . The increase in PRKG1 was significantly $(* P<0.003)$ higher than that in sGC. In $\mathrm{A}-\mathrm{C}$, equal protein loading was controlled by Ponceau staining of the blots.
GSK3A/B (Fig. 6B) and AKT (not shown), representing signaling components of other pathways. In contrast to PRKG1, the levels of these kinases were less affected by EDS treatment and showed decreased rather than increased protein concentrations at day 14 after EDS injection. Interestingly, we identified distinct structural changes in the case of both GSK3 (see the replacement of the band representing GSK3B by a higher molecular weight form) and AKT (not shown) in prostate tissue 14 days after EDS administration.

We then examined whether PRKG1 expression is altered under conditions of Leydig cell depletion also in other androgen-dependent tissues. These studies revealed (Fig. 6B, lower part) that PRKG1 levels do not change in response to androgen withdrawal in penis and epididymis. The absence of treatment effects was evident in both cytosolic and membrane fractions of the epididymis. Thus, these experiments demonstrated a massive and prostate-specific upregulation of PRKG1 in vivo when testosterone supply by Leydig cells is abrogated.

Remarkably, other elements (sGC, NOS3, and PDE5) of cGMP signaling pathways also respond to Leydig cell depletion in the prostate with increased expression levels (Fig. 6C). The extent of elevation of these proteins (ranging from 3.5- to 6.5-fold increases at day 14 after EDS), however, was markedly and significantly lower than that of PRKG1 (12.6-fold).

\section{Discussion}

\section{Organ-specific characteristics of cGMP signaling}

The bladder was characterized by highest expression levels of sGC, NOS3, PDE5, and PRKG1, indicating pivotal local signaling roles for NO/cGMP/cGK pathways. The extraordinary abundance of PDE5 protein (bladder, prostate, epididymis, and testis) among rat reproductive/lower-urinary tract organs correlates with previously published Pde5 gene expression data (Filippi et al. 2007) and marks the bladder as a favorable target for PDE5 inhibitor-based therapeutic interventions. Compared with lung, where high PDE5 provided a molecular basis for treatment of pulmonary hypertension (Corbin et al. 2005), cytosolic PDE5 abundance is similar and membrane concentrations are even higher in the bladder. Immunohistochemical data localized PDE5 to the bladder smooth muscle (Fibbi et al. 2010), and both NO donors and PDE5 inhibitors were found to reduce the muscle tonus in experiments with rat and human bladder tissues (Sandner et al. 2009), although cAMPmediated pathways appear to play a predominant role for direct detrusor muscle relaxation (Andersson \& Arner 2004). Tension release during the bladder filling phase is crucial and requires robust mechanisms to generate smooth muscle relaxation. This kind of relaxation requirement is a specific feature of the bladder and 
may explain basically the exceptional abundance of NOS3, sGC, PDE5, and PRKG1 in this organ. In functional support, PRKG1-deficient mice showed bladder hyperactivity with reduced bladder capacity and micturition volumes (Persson et al. 2000). There is increasing evidence that NO-cGMP signaling also controls sensory pathways of the micturition reflex (Caremel et al. 2010) and is active in interstitial cells to modulate phasic contractile activity (Lagou et al. 2006). Our findings demonstrating predominant expression levels and agonist-induced activity of GC-A (compared with GC-B) suggest additional local roles mainly for ANP-cGMP signaling. In support and indicating possible sites of physiological activity, ANP (but not CNP)responsive cells have been identified by cGMP immunostaining in suburothelial cells of guinea pig bladders (de Vente et al. 2007).

The expression of all cGMP pathway proteins examined was extremely low in the prostate, uncovering a marked attribute of this organ. Indicating not only a tissue-specific but also a signaling route-specific phenomenon, crucial components of other signaling pathways (e.g. GSK3A/B) did not show a reduced expression in this tissue. Our findings are in apparent disagreement with previous data showing high abundance of PDE5 mRNA in the human prostate (Morelli et al. 2004) and question the view that androgendependent upregulation leads to generally enhanced PDE5 expression in organs of the male genital tract (Morelli et al. 2004). Compatible with our results, however, a recent characterization of PDE5 mRNA distribution in human male lower-urinary tract tissues also revealed lowest abundance in the prostate gland (Fibbi et al. 2010). Thus, the weak expression of PDE5 (and probably the other cGMP signaling proteins) in the prostate may not represent a rat-specific phenomenon. The physiological meaning of a minimized predisposition of cGMP pathway proteins in this tissue remains an exciting item.

Although there is evidence for cGMP-mediated relaxing activity in prostate smooth muscle (Kedia et al. 2008), recent findings focused the attention to local roles in the control of cellular proliferation and differentiation (Sandner et al. 2009, Zenzmaier et al. 2010). Pharmacological inhibition of PDE5 reduced the proliferation of primary prostate stromal cells and attenuated fibroblast-to-myofibroblast trans-differentiation, in common, suggesting therapeutic potential for prevention and treatment of benign prostatic hyperplasia (Zenzmaier et al. 2010). Interestingly, these data, indicating pronounced cellular activity under conditions of low PDE5 (and other cGMP pathway proteins), resemble findings in heart tissue. Chronic PDE5 inhibition prevents cardiac hypertrophy (Takimoto et al. 2005a) despite a poor local Pde5 gene expression, which is 100-fold lower than in lung (Takimoto et al. 2005b). Likewise, ANP-cGMP signaling inhibits cardiac hypertrophy (Bubikat et al. 2005), although the ANP receptor, GC-A, is of unusually low abundance in the heart (D Müller, unpublished results). Thus, exiguous expression of PDE5 and cGMP-generating proteins does not preclude crucial growth-regulating effects and their intensification by PDE5 inhibition.

This study shows that the epididymis is distinguished by pronounced expression of membrane GCs (in particular GC-B), although sGC and NOS3 are less abundant than in the bladder. The findings are consistent with and support previously reported roles for NPs in the regulation of spontaneous contractility in smooth muscle cells lining the epididymal duct (Mewe et al. 2006). An apparent predominance of GC-B versus GC-A was confirmed by membrane GC assays, where CNP elicited threefold higher amounts of cGMP than ANP. The data probably explain at a molecular level the higher efficacy of CNP (compared with ANP) in reducing the frequency of spontaneous phasic contractions and affecting the muscle tone in bovine epididymal ducts (Mewe et al. 2006). In addition to these activities operating in the control of sperm movement, recent findings elucidated a pivotal role for cGMP signaling in the epididymal epithelium, serving to regulate proton secretion into the lumen (Shum et al. 2008). The resulting luminal acidification is important for proper sperm maturation. Considering the well-established expression of membrane GC in epithelial (e.g. kidney and intestine) and endothelial cells and their impact for ion transport and permeability (Kuhn 2009, Sabbatini 2009), roles for GC-A and/or GC-B in modulating the luminal milieu along the epididymal duct are well conceivable. In apparent support and raising particular future attention to GC-B, CNP is strongly produced by epithelial cells of the epididymis (Nielsen et al. 2008). Of separate significance, our findings demonstrating an exceptionally poor expression of PDE5 in the epididymis, which is even lower than in the prostate, strongly suggest that cGMP-specific PDEs other than PDE5 play a predominant role for controlling CGMP signals in this organ.

\section{Age-related effects}

The pronounced age-related decrease of PDE5 in the bladder represents a potentially significant novel finding. Interestingly, bladder strips from castrated rats are more sensitive to NO-induced relaxation and less responsive to PDE5 inhibitors, suggesting that androgen deprivation evokes downregulation of PDE5 activity (Filippi et al. 2007). Thus, the age-dependent reduction of bladder PDE5 expression may be a direct local consequence of declined testosterone production by aged Leydig cells (Midzak et al. 2009). As reported previously (Paust et al. 2002), the old (23-24 months) Wistar rats had about threefold lower $(0.76 \pm 0.42 \mathrm{ng} / \mathrm{ml} ; n=67)$ serum testosterone concentrations than their young ( 3 months) counterparts $(2.49 \pm 1.68 \mathrm{ng} / \mathrm{ml} ; n=56)$. 
Since we did not find age-related decreases in bladder cGMP-generating proteins, lowered PDE5 abundance might affect the intensity and duration of local cGMP accumulations. Considering the relevance for regulation of muscle contractility and bladder sensations, impaired (i.e. enhanced) cGMP signaling activity could be implicated in the development of age-related bladder dysfunctions (Zhao et al. 2010). However, in contrast to GC-A, in which age-related changes in enzymatic activity were ruled out, cGMP-mediated activation of PDE5 and desensitization of sGC (Halvey et al. 2009) might compensate the reduced local PDE5 abundance. Therefore, our present findings provoke future studies, where the efficacy of PDE5 inhibition on bladder functions is examined in an age- and androgen statusdependent context.

The identification of increased PRKG1 and unchanged PDE5 levels in the aged prostate shows that cGMP pathway proteins are affected by aging in an organspecific manner. The (patho)physiological consequences of elevated PRKG1 abundance in this tissue remain to be elucidated, but disturbance of proper regulation of proliferation/differentiation processes are conceivable. PRKG1 was localized to stromal areas of the transition zone (Kedia et al. 2008) but is not immunohistochemically detectable in epithelial cells (D Müller, A K Mukhopadhyay, M S Davidoff \& R Middendorff, unpublished results). Thus, PRKG1 is expressed in those areas where androgens are thought to regulate prostate stem cell homeostasis and to maintain a growthquiescent adult prostate gland (Love et al. 2009). As advanced age is the most important risk factor for the development of benign prostatic hyperplasia and prostate cancer (Sampson et al. 2007), the identified agerelated increase in PRKG1 may have pathophysiological significance.

Finally, our findings of aging-dependent decreases in membrane-localized but not cytosolic PRKG1 provide evidence for specifically reduced membrane-associated PRKG1 activities in the aged epididymis. Possible functional consequences include attenuated phosphorylation of cGMP-regulated membrane proteins such as ion channels. Based on the role of cGMP signaling for sperm movement, maturation, and modulation of the epididymal duct fluid, the data raise the possibility that molecular changes localized to the epididymis (Syntin \& Robaire 2001) contribute to the aging-associated diminution of semen volume and sperm motility in healthy men (Eskenazi et al. 2003).

\section{Androgen-dependent effects}

The temporary elimination of Leydig cells in rats by EDS treatment represents a valuable in vivo model to assess androgen-dependent effects (Molenaar et al. 1985). We have previously used this model to identify vascular stem/progenitor cells as origin of the new Leydig cell population that emerges about 3 weeks after the EDSinduced cell elimination (Davidoff et al. 2004, 2009). In this study, we used the EDS model to examine the expression of cGMP pathway proteins in the prostate under conditions of androgen withdrawal and re-appearance.

The elucidated massive upregulation of PRKG1 in response to Leydig cell depletion and its complete repression by subsequent endogenous testosterone supplementation identifies PRKG1 as an androgenregulated protein. Importantly, this regulation takes place in prostate but not in other (epididymis and penis) androgen-dependent tissues examined, indicating prostate-specific activity. Recent investigations, using a human prostate xenograft model to characterize a collection of androgen-related genes, revealed up to fivefold gene expression changes in response to either androgen withdrawal or supplementation in mice with the unique exception of a 14-fold change in the case of prostate-specific antigen (Love et al. 2009). In this regard, the 12.6-fold change observed for PRKG1 in our study indicates an exceptionally efficacious regulation and marks PRKG1 as a potential key androgenresponsive signaling element in the prostate. Elevated expression (ranging from 3.5- to 6.5-fold increases) in response to Leydig cell depletion was also observed for sGC, NOS3, and PDE5. The latter data corroborate that PDE5 is not, unlike previously hypothesized (Morelli et al. 2004), generally upregulated by androgens in organs of the male genital tract. More importantly, these findings suggest that suppression of PRKG1 (and other CGMP pathway proteins) represents a mechanism that contributes to an androgen-controlled balance between proliferation, differentiation, and apoptosis in the adult prostate (Sampson et al. 2007). As observed by investigations addressing the molecular mechanisms implicated in vascular remodeling, PRKG1 appears to induce predominantly growth-promoting rather than antiproliferative effects (Alderton et al. 2001). Interestingly, recent findings demonstrated that PRKG1 activity is also not involved in the inhibition of cardiac hypertrophy (Lukowski et al. 2010). Thus, age-related diminution of androgen levels may be causative for the observed increase in prostatic PRKG1 expression and in turn favor cellular proliferation processes. In this regard, it will be of particular interest to compare PRKG1 abundance between normal and hyperplastic prostate tissue and to examine cellular PRKG1 expression in androgen-sensitive and androgen-refractory prostate cancer.

In conclusion, the local abundance of cGMP pathway proteins in male genitourinary tract organs shows remarkable tissue-specific characteristics. Distinct agerelated changes, identified in bladder, prostate, and epididymis, and a striking androgen-dependent regulation in the prostate suggest potential significance for the development of aging-associated malfunctions. 
If the data obtained in rat similarly apply to human tissue, the present findings may provide valuable information for therapeutic interventions with PDE5 inhibitors and/or other agents affecting cGMP signaling pathways.

\section{Materials and Methods}

\section{Animals and tissues}

Male Wistar rats (Charles River Laboratories, Sulzbach, Germany) of two age groups (young $=3$ months and old= 23-24 months) were dissected after decapitation. Tissues (prostate tissue contained ventral, dorsal, and lateral lobes) were frozen in liquid nitrogen and stored at $-80^{\circ} \mathrm{C}$. As specified before (Davidoff et al. 2004), 3-month-old Wistar rats were used to induce Leydig cell depletion by single i.p. injections of EDS ( $75 \mathrm{mg} / \mathrm{kg}$ body weight). The rats were killed 1-47 days after injection, and tissues were handled as described above. All animals were used according to government principles regarding the care and use of animals with permission (G8151/591-00.33) of the local regulatory authority.

\section{Protein preparations}

Frozen tissues were pulverized with addition of liquid nitrogen in a mortar, suspended in $10 \mathrm{ml} / \mathrm{g}$ tissue of ice-cold homogenization buffer (Müller et al. 2002) and homogenized by 15-20 strokes in a Potter-Elvehjem homogenizer. After centrifugation at $3000 \mathrm{~g}$ at $4{ }^{\circ} \mathrm{C}$ for $8 \mathrm{~min}$ to remove debris and nuclei, the supernatant fractions were re-centrifuged at $4{ }^{\circ} \mathrm{C}$ for $30 \mathrm{~min}$ at $100000 \mathrm{~g}$. Supernatants were collected and used as cytosolic protein fractions. The pellets were re-suspended in suitable volumes of $50 \mathrm{mM}$ Tris- $\mathrm{HCl}(\mathrm{pH} \mathrm{7.5)}$ to attain membrane protein concentrations of $2-6 \mu \mathrm{g} / \mu \mathrm{l}$. Proteins were stored at $-80^{\circ} \mathrm{C}$, and concentrations were determined using a kit from Bio-Rad with BSA as standard.

\section{Immunoblotting}

After boiling for $3 \mathrm{~min}$ in SDS-PAGE sample buffer (Müller et al. 2002), proteins were resolved by SDS-PAGE under reducing conditions and transferred to nitrocellulose membranes (Müller et al. 2004). Blots were stained with Ponceau S (Sigma), and positions of co-migrated reference proteins (SDS-6H, Sigma) were marked using a black ball-pen. Prior to de-staining in $\mathrm{H}_{2} \mathrm{O}$, records of the images were derived by scanning and served to control protein loading. Blots were pre-treated with blocking solution as described before (Müller et al. 2004, 2010a). Polyclonal antibodies against GC-A (PGCA-101AP, FabGennix, Frisco, TX, USA), GC-B (PGCB-201AP, FabGennix), sGC 31 (CM160897, IBL, Hamburg, Germany), PDE5 (\#4072; Cell Signaling, Beverly, MA, USA), PRKG1 (PK1018; Calbiochem, Nottingham, UK), GSK3A/B (KAM-ST002E; Stressgen, Victoria, Canada), PTEN (\#07-1372; Millipore, Schwalbach, Germany), AKT (\#07-1643, Millipore), CYP11A1 (AB1244; Chemicon, Temecula, CA, USA), or MABs against NOS3 (N30020; Transduction, San Jose, CA, USA) were used. Goat anti-rabbit or anti-mouse IgG
(Pierce, Rockford, IL, USA), linked to peroxidase, served as secondary antibodies. Signals were detected via ECL (Müller et al. 2004). After stripping (Müller et al. 2010a), blots were re-probed consecutively with other antibodies.

\section{Quantitative immunoblot analyses and densitometric measurements}

For quantitative evaluations (up to 13), protein samples were applied in equal volumes (ranging from 30 to $70 \mu \mathrm{l}$ ) to the same gel. After SDS-PAGE and blotting, loading of equal protein amounts was controlled by Ponceau staining (see Supplementary Fig. 4). This approach was found to provide several advantages over the frequently used immunodetection of proteins such as glyceraldehyde 3-phosphate dehydrogenase (GAPDH) or actin as loading control. Relevant points: 1) inspection of the protein staining easily detects (prior to antibody exposures) lanes where anomalous protein amounts have been loaded. Such blots were not used for quantitative evaluations, and protein concentrations of the samples in question were re-determined. 2) As all tissues/organs provide a specific pattern of abundantly expressed proteins, assessment of protein staining proves that extracts of the same organs have been loaded. More importantly, it allows controlling the homogeneity of tissue dissection. By this, we were able to identify (and discard) single samples, containing apparently significant amounts of unrelated (surrounding) tissue. 3) Protein staining (but not GAPDH detection) controls the proper transfer of proteins to the membrane on the whole blot. 4) GAPDH mRNA expression varies up to 15-fold among different tissue types (Barber et al. 2005). Thus, using GAPDH levels for normalization across different tissues (as was required in the case of Fig. 1C) may generate misleading results. 5) It facilitates electrophoretic separation under conditions (e.g. acrylamide concentrations and running times), which are most appropriate for band resolution of the target protein (an important issue for subsequent densitometric evaluations). Under such conditions, small proteins like GAPDH (36 kDa) have frequently migrated out of the gel, preventing their usage as loading control.

To obtain suitable band intensities, different antibody dilutions and/or loaded protein amounts were tested. To prove that primary antibodies properly recognized differences in the level of their target proteins, various defined protein amounts of the same sample were analyzed (see Fig. 3A, which also demonstrates the reliability of Ponceau staining as loading control). After incubation of blots with chemiluminescent reagent, images with varying band intensities were derived by successive exposure to X-ray films for different time periods. One final exposition overnight served to control that the chemiluminescent reagent was spread evenly over the membrane. Films appropriate for densitometry were scanned (Epson Perfection V 700 Photo; Meerbusch, Germany) using the settings standard mode, photo, and grayscale at a resolution of 300 dpi. Images were saved as TIFF files. These were imported for densitometric quantifications using Image 1.42 software (open source; NIH, Bethesda, MD, USA). Bands were selected as demonstrated in Supplementary Fig. 4 and peak areas were analyzed. To combine data sets from 
different immunoblots, densitometry scores were normalized. Finally, all values were reduced proportionally to attain single-digit units.

\section{Membrane GC assays}

The procedure of membrane GC assays has been reported (Müller et al. 2004). In brief, membranes (10 $\mu$ g protein) were incubated in either the absence or the presence of NPs for $12 \mathrm{~min}$ at $37^{\circ} \mathrm{C}$. The amounts of CGMP generated were measured by ELISA as described (Müller et al. 2004).

\section{Data analysis}

Data were graphed and analyzed using Prism 3.02 (GraphPad Software, Inc., San Diego, CA, USA). The significance of effects was assessed using Student's $t$-test.

\section{Supplementary data}

This is linked to the online version of the paper at http://dx.doi. org/10.1530/REP-10-0517.

\section{Declaration of interest}

The authors declare that there is no conflict of interest that could be perceived as prejudicing the impartiality of the research reported.

\section{Funding}

This work was supported by grants from the Deutsche Forschungsgemeinschaft (SFB 547 C13; KFO 181/1) and Bundesministerium für Forschung und Technologie (01 KY 9103/0).

\section{Acknowledgements}

We thank Jörn Lübberstedt for excellent technical assistance.

\section{References}

Alderton WK, Cooper CE \& Knowles RG 2001 Nitric oxide synthases: structure, function and inhibition. Biochemical Journal 357 593-615. (doi:10.1042/0264-6021:3570593)

Andersson KE \& Arner A 2004 Urinary bladder contraction and relaxation: physiology and pathophysiology. Physiological Reviews 84 935-986. (doi:10.1152/physrev.00038.2003)

Andersson KE, Uckert S, Stief C \& Hedlund C 2007 Phosphodiesterases (PDEs) and PDE inhibitors for treatment of LUTS. Neurourology and Urodynamics 26 928-933. (doi:10.1002/nau.20485)

Barber RD, Harmer DW, Coleman RA \& Clark BJ 2005 GAPDH as a housekeeping gene: analysis of GAPDH mRNA expression in a panel of 72 human tissues. Physiol Genomics 21 389-395. (doi:10.1152/ physiolgenomics.00025.2005)

Bubikat A, De Windt LJ, Zetsche B, Fabritz L, Sickler H, Eckardt D, Gödecke A, Baba HA \& Kuhn M 2005 Local atrial natriuretic peptide signaling prevents hypertensive cardiac hypertrophy in endothelial nitricoxide synthase-deficient mice. Journal of Biological Chemistry 280 21594-21599. (doi:10.1074/jbc.M501103200)
Caremel R, Oger-Roussel S, Behr-Roussel D, Grise P \& Giuliano FA 2010 Nitric oxide/cyclic guanosine monophosphate signaling mediates an inhibitory action on sensory pathways of the micturition reflex in the rat. European Urology 58 616-625. (doi:10.1016/j.eururo.2010.07.026)

Corbin JD, Beasley A, Blount MA \& Francis SH 2005 High lung PDE5: a strong basis for treating pulmonary hypertension with PDE5 inhibitors. Biochemical and Biophysical Research Communications 334 930-938. (doi:10.1016/j.bbrc.2005.06.183)

Davidoff MS, Middendorff M, Enikolopov G, Riethmacher D, Holstein AF \& Müller D 2004 Progenitor cells of the testosterone-producing Leydig cells revealed. Journal of Cell Biology 167 935-944. (doi:10.1083/jcb. 200409107)

Davidoff MS, Middendorff R, Müller D \& Holstein AF 2009 The neuroendocrine Leydig cells and their stem cell progenitors, the pericytes. Advances in Anatomy, Embryology, and Cell Biology 205 $1-107$.

Dimitriadis F, Tsounapi P, Saito M, Watanabe T, Sylakos A, Tsabalas S, Miyagawa I \& Sofikitis N 2009 Is there a role for PDE5 inhibitors in the management of male infertility due to defects in testicular or epididymal function? Current Pharmaceutical Design 15 3506-3520. (doi:10.2174/ 138161209789207015)

Domek-topacińska KU \& Strosznajder JB 2010 Cyclic GMP and nitric oxide synthase in aging and Alzheimer's disease. Molecular Neurobiology 41 129-137. (doi:10.1007/s12035-010-8104-x)

Eskenazi B, Wyrobek AJ, Sloter E, Kidd SA, Moore L, Young S \& Moore D 2003 The association of age and semen quality in healthy men. Human Reproduction 18 447-454. (doi:10.1093/humrep/deg107)

Fibbi B, Morelli A, Vignozzi L, Filippi S, Chavalmane A, De Vita G, Marini M, Gacci M, Vannelli GB, Sandner P et al. 2010 Characterization of phosphodiesterase type 5 expression and functional activity in the human male lower urinary tract. Journal of Sexual Medicine 7 59-69. (doi:10.1111/j.1743-6109.2009.01511.x)

Filippi S, Morelli A, Sandner P, Fibbi B, Mancina R, Marini M, Gacci M, Vignozzi L, Vannelli GB, Carini M et al. 2007 Characterization and functional role of androgen-dependent PDE5 activity in the bladder. Endocrinology 148 1019-1029. (doi:10.1210/en.2006-1079)

Ghofrani HA, Osterloh IH \& Grimminger F 2006 Sildenafil: from angina to erectile dysfunction to pulmonary hypertension and beyond. Nature Reviews. Drug Discovery 5 689-702. (doi:10.1038/nrd2030)

Goldstein AS, Huang J, Guo C, Garraway IP \& Witte ON 2010 Identification of a cell of origin for human prostate cancer. Science 329 568-571. (doi:10.1126/science.1189992)

Gradini R, Realacci M, Ginepri A, Naso G, Santangelo C, Cela O, Sale P, Berardi A, Petrangeli E, Gallucci M et al. 1999 Nitric oxide synthases in normal and benign hyperplastic human prostate: immunohistochemistry and molecular biology. Journal of Pathology 189 224-229. (doi:10.1002/ (SICl)1096-9896(199910)189:2 <224::AID-PATH422 > 3.0.CO;2-K)

Halvey EJ, Vernon J, Roy B \& Garthwaite J 2009 Mechanisms of activitydependent plasticity in cellular nitric oxide-cGMP signaling. Journal of Biological Chemistry 284 25630-25641. (doi:10.1074/jbc.M109.030 338)

Hofmann F, Feil R, Kleppisch T \& Schlossmann J 2006 Function of cGMPdependent protein kinases as revealed by gene deletion. Physiological Reviews 86 1-23. (doi:10.1152/physrev.00015.2005)

Kedia GT, Uckert S, Jonas U, Kuczyk MA \& Burchardt M 2008 The nitric oxide pathway in the human prostate: clinical implications in men with lower urinary tract symptoms. World Journal of Urology 26 603-609. (doi:10.1007/s00345-008-0303-y)

Kuhn M 2009 Function and dysfunction of mammalian membrane guanylyl cyclase receptors: lessons from genetic mouse models and implications for human diseases. Handbook of Experimental Pharmacology 191 47-69. (doi:10.1007/978-3-540-68964-5_4)

Lagou M, Drake MJ, Markerink-van Ittersum M, De Vente J \& Gillespie JI 2006 Interstitial cells and phasic activity in the isolated mouse bladder. British Journal of Urology International 98 643-650. (doi:10.1111/ j.1464-410X.2006.06255.x)

Lang SH, Frame FM \& Collins AT 2009 Prostate cancer stem cells. Journal of Pathology 217 299-306. (doi:10.1002/path.2478)

Love HD, Booton SE, Boone BE, Breyer JP, Koyama T, Revelo MP, Shappell SB, Smith JR \& Hayward SW 2009 Androgen regulated genes in human prostate xenografts in mice: relation to $\mathrm{BPH}$ and prostate cancer. PLOS ONE 4 e8384. (doi:10.1371/journal.pone.0008384) 
Lukowski R, Rybalkin SD, Loga F, Leiss V, Beavo JA \& Hofmann F 2010 Cardiac hypertrophy is not amplified by deletion of cGMP-dependent protein kinase I in cardiomyocytes. PNAS 107 5646-5651. (doi:10.1073/ pnas.1001360107)

Mewe M, Bauer CK, Müller D \& Middendorff R 2006 Regulation of spontaneous contractile activity in the bovine epididymal duct by cyclic guanosine 5'-monophosphate-dependent pathways. Endocrinology 147 2051-2062. (doi:10.1210/en.2005-1324)

Midzak AS, Chen H, Papadopoulos V \& Zirkin BR 2009 Leydig cell aging and the mechanisms of reduced testosterone synthesis. Molecular and Cellular Endocrinology 299 23-31. (doi:10.1016/j.mce.2008.07.016)

Molenaar R, de Rooij DG, Rommerts FF, Reuvers PJ \& van der Molen HJ 1985 Specific destruction of Leydig cells in mature rats after in vivo administration of ethane dimethyl sulfonate. Biology of Reproduction 33 1213-1222. (doi:10.1095/biolreprod33.5.1213)

Morelli A, Filippi S, Mancina R, Luconi M, Vignozzi L, Marini M, Orlando C, Vannelli GB, Aversa A, Natali A et al. 2004 Androgens regulate phosphodiesterase type 5 expression and functional activity in corpora cavernosa. Endocrinology 145 2253-2263. (doi:10.1210/en.2003-1699)

Müller D, Middendorff R, Olcese J \& Mukhopadhyay AK 2002 Central nervous system-specific glycosylation of the type A natriuretic peptide receptor. Endocrinology 143 23-29. (doi:10.1210/en.143.1.23)

Müller D, Mukhopadhyay AK, Speth RC, Guidone G, Potthast R, Potter LR \& Middendorff $\mathbf{R} 2004$ Spatiotemporal regulation of the two atrial natriuretic peptide receptors in testis. Endocrinology 145 1392-1401. (doi:10.1210/en.2003-0706)

Müller D, Greenland KJ, Speth RC \& Middendorff R 2010a Neuronal differentiation of NG108-15 cells has impact on nitric oxide- and membrane (natriuretic peptide receptor-A) cyclic GMP-generating proteins. Molecular and Cellular Endocrinology 320 118-127. (doi:10. 1016/j.mce.2010.01.022)

Müller D, Hildebrand M, Lübberstedt J, Kuhn M \& Middendorff R $2010 \mathrm{~b}$ The membrane receptors guanylyl cyclase-A (GC-A) and GC-B undergo distinctive changes in posttranslational modification during brain development. Journal of Neurochemistry 115 1024-1034. (doi:10. 1111/j.1471-4159.2010.06985.x)

Nielsen SJ, Gøtze JP, Jensen HL \& Rehfeld JF 2008 ProCNP and CNP are expressed primarily in male genital organs. Regulatory Peptides $\mathbf{1 4 6}$ 204-212. (doi:10.1016/j.regpep.2007.09.022)

Paust HJ, Wessels J, Ivell R \& Mukhopadhyay AK 2002 The expression of the RLF/INSL3 gene is reduced in Leydig cells of the aging rat testis. Experimental Gerontology 37 1461-1467. (doi:10.1016/S0531-5565(02) 00098-0)

Persson K, Pandita RK, Aszòdi A, Ahmad M, Pfeifer A, Fässler R \& Andersson K-E 2000 Functional characteristics of urinary tract smooth muscles in mice lacking cGMP protein kinase type I. American Journal of Physiology. Regulatory, Integrative and Comparative Physiology 279 R1112-R1120.

Sabbatini ME 2009 Natriuretic peptides as regulatory mediators of secretory activity in the digestive system. Regulatory Peptides 154 5-15. (doi:10. 1016/j.regpep.2009.02.009)
Sampson N, Untergasser G, Plas E \& Berger P 2007 The ageing male reproductive tract. Journal of Pathology 211 206-218. (doi:10.1002/ path.2077)

Sandner P, Neuser D \& Bischoff E 2009 Erectile dysfunction and lower urinary tract. Handbook of Experimental Pharmacology 191 507-531.

Shum WW, Da Silva N, McKee M, Smith PJ, Brown D \& Breton S 2008 Transepithelial projections from basal cells are luminal sensors in pseudostratified epithelia. Cell 135 1108-1117. (doi:10.1016/j.cell. 2008.10.020)

Stice JP, Eiserich JP \& Knowlton AA 2009 Role of aging versus the loss of estrogens in the reduction in vascular function in female rats. Endocrinology 150 212-219. (doi:10.1210/en.2008-0640)

Syntin P \& Robaire B 2001 Sperm structural and motility changes during aging in the Brown Norway rat. Journal of Andrology 22 235-244.

Takimoto E, Champion HC, Li M, Belardi D, Ren S, Rodriguez ER, Bedja D, Gabrielson KL, Wang Y \& Kass DA 2005a Chronic inhibition of cyclic GMP phosphodiesterase 5A prevents and reverses cardiac hypertrophy. Nature Medicine 11 214-222. (doi:10.1038/nm1175)

Takimoto E, Champion HC, Belardi D, Moslehi J, Mongillo M, Mergia E, Montrose DC, Isoda T, Aufiero K, Zaccolo M et al. 2005b cGMP catabolism by phosphodiesterase $5 \mathrm{~A}$ regulates cardiac adrenergic stimulation by NOS3-dependent mechanism. Circulation Research $\mathbf{9 6}$ 100-109. (doi:10.1161/01.RES.0000152262.22968.72)

Takimoto E, Koitabashi N, Hsu S, Ketner EA, Zhang M, Nagayama T, Bedja D, Gabrielson KL, Blanton R, Siderovski DP et al. 2009 Regulator of $\mathrm{G}$ protein signaling 2 mediates cardiac compensation to pressure overload and antihypertrophic effects of PDE5 inhibition in mice. Journal of Clinical Investigation 119 408-420. (doi:10.1172/ JCl35620)

de Vente J, Markerink-van Ittersum M \& Gillespie JI 2007 Natriuretic peptide responsive, cyclic guanosine monophosphate producing structures in the guinea pig bladder. Journal of Urology 177 1191-1194. (doi:10.1016/j.juro.2006.10.039)

Wang C 2010 Phosphodiesterase-5 inhibitors and benign prostatic hyperplasia. Current Opinion in Urology 20 49-54. (doi:10.1097/MOU. 0b013e328333ac68)

Zenzmaier C, Sampson N, Pernkopf D, Plas E, Untergasser G \& Berger P 2010 Attenuated proliferation and trans-differentiation of prostatic stromal cells indicate suitability of phosphodiesterase type 5 inhibitors for prevention and treatment of benign prostatic hyperplasia. Endocrinology 151 3975-3984. (doi:10.1210/en.2009-1411)

Zhao W, Aboushwareb T, Turner C, Mathis C, Bennett C, Sonntag WE, Andersson K-E \& Christ G 2010 Impaired bladder function in aging male rats. Journal of Urology 184 378-385. (doi:10.1016/j.juro.2010.03.004)

Received 15 December 2010

First decision 3 February 2011

Revised manuscript received 29 March 2011

Accepted 21 April 2011 